Research Paper:

\title{
Effects of a Care Package on the Shelf Life and Incidence of the Infection of Central Peripheral Venous Catheter in the Premature Infants Admitted to the NICU
}

\author{
Fariba Safaee $^{1}$, Mohammad Kazemian² (D), Leili Borimnejad ${ }^{3 *}$ (D), Marjan Rasouli
}

1. Department of Pediatric Nursing, School of Nursing and Midwifery, Iran University of Medical Sciences, Tehran, Iran.

2. Department of Neonatology, Neonatal Health Research Center, School of Medicine, Mofid Children's Hospita, Shahid Beheshti University of Medical Sciences, Tehran, Iran.

3. Nursing Care Research Center, Iran University of Medical Sciences, Tehran, Iran.

4. Department of Nursing, Faculty of Medicine, Qom Branch, Islamic Azad University, Qum, Iran.

\begin{tabular}{|c|c|}
\hline $\begin{array}{l}\text { Use your device to scan } \\
\text { and read the article online }\end{array}$ & Cltation: Safaee, F., et al., 2020. Effects of a Care Package on the Shelf Life and Incidence of the Infection of Central Pe- \\
\hline Q & $\begin{array}{l}\text { ripheral Venous Catheter in the Premature Infants Admitted to the NICU. Journal of Client-Centered Nursing Care, 6(4), pp. } \\
\text { 231-238. https://doi.org/10.32598/JCCNC.6.4.169.7 }\end{array}$ \\
\hline aring & dol'https://doi.org/10.32598/JCCNC.6.4.169.7 \\
\hline
\end{tabular}

\section{(1) (\$)}

Article info:

Received: 23 Jul 2020

Accepted: 07 Aug 2020

Published: 01 Nov 2020
Keywords:

Central vein, Infection, Neonatal, Critical care, Peripheral catheterization

\begin{abstract}
A B S T RA C T
Background: Neonates require continuous and reliable intravenous routes to receive fluids, intravenous nutrition, and medications; accordingly, repeated interventions are essential to implant these routes. Percutaneous catheterization is a technology used for this purpose. Considering that central line infections are a major concern in Neonatal Intensive Care Units (NICUs), the present study aimed to assess the effects of using a care package on the incidence of infections and shelf life of peripheral central catheterization in the premature infants admitted to the NICU.

Methods: This quasi-experimental study was a process assessment research conducted on 131 premature catheterized infants who were admitted to the NICU of Mofid Children's Hospital affiliated to Shahid Beheshti University of Medical Sciences, Tehran, Iran, in 2019. The study sample included 131 neonates who met the inclusion criteria. The neonates were enrolled in the study at two levels, including 53 subjects in the first four months before the intervention, and 78 in the second four months after application of the care package. The infants were selected via the convenience sampling method. Data were collected using the central line maintenance bundle and central line maintenance bundle daily checklists. The catheter insertion method and the degree of catheter tip infection were evaluated before and after the protocol training program for the nurses. The results of catheter tip culture after removal were considered as the training outcomes. Data analysis was performed in SPSS V. 16, using descriptive statistics, independent $t$ test, the Fisher exact test, and the chi-squared test.

Results: The results of the catheter tip culture confirmed the presence of pathogens in the catheter and culture of 18 samples (34\%), before the intervention, while a reduction was denoted in 14 samples $(17.9 \%)$ after the intervention; the reduction was statistically significant $(\mathrm{P}=0.036)$. Furthermore, the shelf life of the catheter increased after the intervention, however, the difference was not significant.

Conclusion: According to the results, applying the care package reduces the incidence of central peripheral venous catheter infection in neonates admitted to the NICU. Therefore, it is suggested to incorporate this package into the care instructions of neonatal wards.
\end{abstract}

\footnotetext{
* Corresponding Author:

Leili Borimnejad, PhD.

Address: Nursing Care Research Center, Iran University of Medical Sciences, Tehran, Iran

Tel: +98 (912) 5029548

E-mail: borimnejad.l@Iums.ac.ir
} 


\section{Highlights}

- Evidence-based strategies recommend the application of a care package to reduce central catheter infection in NICUs.

- The present study investigated the effects of applying a care package on the incidence of infection and the shelf life of central peripheral venous catheters, in the premature infants admitted to the NICU.

- The obtained results indicated that the standard guideline training of nurses could reduce the incidence of central venous catheter infection in infants.

\section{Plain Language Summary}

Low-birth-weight infants often require intravenous access for more than six days. Therefore, a central peripheral venous catheter is considered to be an acceptable alternative to provide long-term access to the vein with few complications and no restrictions on any injections. The nurses who are involved in the implantation of central peripheral venous catheters in infants and children must have sufficient knowledge and experience in the care of this population. The present study aimed to assess the effects of using a care package on the incidence of infections and the shelf life of central peripheral venous catheterization in the premature infants admitted to the NICUs. According to the obtained results, the standard guideline training of nurses reduces the incidence of central venous catheter infection in infants.

\section{Introduction}

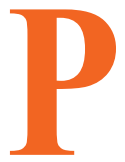

remature birth is one of the leading causes of annual infant mortality. Therefore, it is necessary to solve the problems related to premature infants, using appropriate measures. Scientific evidence attests to the prolonged hospitalization of infants in developing countries (Santhakumaran et al., 2018). As infants are admitted to the intensive care unit, emphasis should be placed on their intravenous access methods, since these infants typically require intravenous nutrition. Besides, the amount and composition of the prescribed fluids may widely vary, considering that the renal hemostatic mechanisms may be immature, and the mortality rate associated with electrolyte and acid-base disorders is higher in these infants. Consequently, these infants require continuous and reliable intravenous routes to receive fluids, intravenous nutrition, and medications. However, in infants, it is rather difficult to maintain peripheral intravenous accesses and their shelf life is also short. Also, premature infants need repeated interventions to implant the catheters. Nowadays, percutaneous catheterization is commonly used for this purpose, as it is simple and has high resistance and durability (Wang et al., 2015).

Generally, percutaneous catheterization should be considered when intravenous access is required for more than six days. Since Low-Birth-Weight (LBW) infants typically require intravenous access for more than six days, a Peripherally Inserted Central Catheter (PICC) is considered to be an acceptable alternative to provide long-term access to the vein with few complications and no restrictions on the infusion of solutions. Since 1970, peripheral central venous catheterization has been the most appropriate approach to the safe and long-term management of access to the venous routes (Cotogni et al., 2015). However, catheter implantation is associated with complications, such as infection, thrombosis, and embolism, which could increase perforation and mortality (Baranowski, Hummler \& Hopfner 2014). According to reports, central line-associated bloodstream infection is the most costly and life-threatening complication caused by venous catheterization, in Europe, accounting for $10 \%$ to $20 \%$ of nosocomial infections and causative organisms, such as the natural skin flora (Wang et al., 2015). Central line circulatory infections are a grave concern in Neonatal Intensive Care Units (NICUs). Improper catheter implantation could lead to a wide range of complications, such as various infections and increased mortality. Thus, it is imperative to identify effective approaches to the reduction and elimination of these infections, as LBW infants mostly have a poor immune response and may not show specific symptoms after developing an infection (Wang et al., 2015).

In Iran, no official statistics are available on the circulatory infections associated with the central line and central venous catheterization through the skin in infants. In other countries, highly qualified nursing teams are respon- 
sible for the insertion, monitoring, and maintenance of PICCs. According to the principles of the Infusion Nurses Society (INS), these procedures should be performed by nurses who have formal qualifications and have been trained on the insertion of PICC (Gorski, 2017). As the supervisors and managers of treatment teams, physicians often provide written instructions for PICC implantation, then, nurses perform PICC implantation interventions. The priority of the INS in this treatment approach is comprehensive patient support, and only the INS standards could provide the legal principles for intravenous interventions (Alexandrou et al., 2018). Besides, these standards could be used in legal courts for any issues related to these interventions and are ranked first, compared with the principles and views of other institutions and foundations, such as the Center for Disease Control, National Association of Neonatal Nurses, and American Vocational Association, which also provide legal documentation for intravenous interventions (Rosenthal et al., 2020).

Nurses involved in the insertion of PICCs in infants and children should have sufficient knowledge and experience for the proper care of this population, as PICC implantation is rather difficult in infants. All the fifty states of the United States have specific and focused training programs for nurses on PICC implantation. These programs encompass a thorough understanding of the venous anatomy, the selection of the appropriate vein, catheter insertion methods, and catheter care and maintenance. Moreover, recent technologies and innovations have improved the safety of central catheters. Examples of these innovations include the central venous care package or the clinical behaviors of the treatment team, which can reduce the rate of complications to zero if performed concurrently and accurately. These packages should also be simple and scalable based on evidence-based findings. Care packages with strict adherence to handwashing protocols provide a detailed training program on skin disinfection and catheterization procedures (Emoli et al., 2014).

Evidence-based scientific strategies highly recommend the use of care packages to reduce central catheter infection in NICUs. However, further research is required to ensure that such care packages could effectively decrease central catheter infections. Also, the hospital policy of moderate adherence to package administration may not adequately reduce central catheter infections. Reducing infections is only possible by $95 \%$ or more adherence to the practice; despite the knowledge of care in this regard, its application remains challenging (Gilbert et al., 2019).

Current studies and scientific evidence on the infections and other complications of PICC and the preven- tive methods of these cases highlight the importance of nurses' awareness of standard methods to minimize these complications. However, scientific evidence is scarce, regarding the complications caused by PICC. Therefore, data should be obtained to implement proper strategies for the improvement of patient safety, reducing healthcare costs, and decreasing the incidence of PICCrelated infections, which in turn bridge the knowledge gap. Given this knowledge gap, the present study aimed to determine the effects of using a care package on the incidence of infections and the shelf life of PICCs in the premature infants admitted to the NICU.

\section{Materials and Methods}

\section{Design}

This quasi-experimental study as a process assessment research was conducted on the neonates admitted to the NICU. The ranges of gestational age and the birth weight of the neonates were 32 to 36 weeks and 1500 to 2500 grams, respectively. The subjects were examined at two intervals before and after the presentation of the care package, then, the outcomes of the study groups were compared.

\section{Setting}

The study was performed at the NICU of Mofid Children's Hospital affiliated to Shahid Beheshti University of Medical Sciences, Tehran, Iran, in 2019. Mofid Children's Hospital is a referral center, the NICU of which has 31 active beds; the infants requiring level III care are admitted to this ward. In this ward, the mean length of the hospital stay of the neonates is 17 days. In this section, a permanent team of five members is responsible for PICC insertion. We selected this setting for our research, given the importance and necessity of familiarizing the hospital staff with the proper insertion and care of PICC and the use of the care package.

\section{Sample}

The study sample consisted of 131 infants. The inclusion criteria were as follows: 1. PICC implantation for the first time; 2. Negative blood culture; 3. No clinical signs of sepsis; and 4. No congenital anomalies. If premature sepsis developed and symptoms appeared 1 to 3 days after birth, the infant would be excluded from the study. In total, 53 neonates were examined within the first four months, before the intervention, then, 78 infants were evaluated within the second four months, after the care package implementation. 


\section{Data collection}

The data were collected using two checklists: a standard checklist for recording the accurate scrub and aseptic techniques and a catheter daily care checklist. The latter was introduced by the Center for Disease Control, in 2011, and the former has been designed to implement a care package with 16 questions on the demographic characteristics of the neonate; the monitoring of hand hygiene, wearing gloves, masks, hats, and glasses; and catheter insertion. This checklist was used during catheterization in the present study. The second checklist included 10 questions to diagnose and classify the infection daily. For the first time in Iran, this tool was translated to be applied in the current research.

Per the approved protocols, translation was carried out by two translators, then, two other translators who were fluent in English and Persian retranslated the tool. Next, three faculty members reviewed the Persian version, the original version, and the written explanations about the objectives of the study and research methodology. After obtaining their comments, the necessary corrections were made, considering the guidance of the experts. Since this tool had not been used before in the NICUs in Iran, the simultaneous observation method was used to determine the reliability and internal consistency of the instrument. Under similar circumstances, the checklist was completed by two trained individuals; the outcomes were the same with no difference in all the cases, therefore, no test was required. To calculate the scores, the frequency and percentage of yes and no responses were compared in both groups, before and after the intervention. Besides, the infant profile form was completed for the subjects; the form included data on the demographic profile and disease status.

\section{Procedure}

Data collection was initiated after obtaining the required permit. Based on the medical records and using the hospital computer registration system, we reviewed and documented the laboratory information of the medical records of 53 infants with a history of PICC implantation, considering the inclusion criteria of the study during four months before starting the treatment (March-June 2017). To implement the training program, the necessary coordination was made with the head of the department and its staff. Then, the researcher implemented the training program for the insertion team, in coordination with all the department staff. This five-member team was qualified to insert a central peripheral venous catheter and had passed the necessary test. The training program was a three-hour workshop on the implementation of the care package and the insertion and maintenance of PICC. The training program included one hour of the theoretical training of the care package instructions, complying with its five keys during catheterization (hand hygiene, maximum protection precautions while implanting a catheter, skin disinfection with chlorhexidine and iodine, the proper selection of the catheter site, the accurate measurement of the catheter length for implantation, and the daily inspection of the catheter and quick removal of an unnecessary catheter); how to use checklists during catheterization; daily catheter care; and the methods of sending a culture from the catheter to the laboratory. Moreover, the intervention was provided in the form of a workshop in two days and included two hours of practical training to implement the care package program in three catheterization cases. After evaluating the skills of the personnel, resolving the problems, answering the questions, and ensuring the correct implementation of the care package, the trained team inserted the catheter in a trained manner; this stage of the study was performed from March to June 2018. In this process, the nurses who were in charge of catheter insertion in the morning shift implanted all the catheters by observing the standard checklists during catheterization, also, the daily checklist was used in all the shifts until catheter removal. Catheter care was also evaluated and recorded in a special form. The insertion team and the research assistant attended the ward in the fixed morning shifts. Besides, the researcher recorded catheter care in the evening and night shifts, based on the self-report of the clinical nurses.

At the catheter removal, the same trained team collected the culture from the catheter tip in a sterile manner. After wearing sterile gloves and removing the catheter, five centimeters of the catheter tip was placed in a sterile culture dish and transferred to the laboratory. In total, seven samples were found to be infected during sampling and were discarded. In the second period after the intervention, 78 samples were collected within four months. The shelf life of the catheter was also evaluated as the dependent variable, at the time of removal. Thus, the catheter was inserted, the dressing was changed, and the culture was obtained from the catheter tip, only in the morning shift under the supervision of the insertion team and the researcher.

For catheterization per the mentioned five keys, single branch catheters (sizes 1 and 2) were selected based on the age and weight of the infant. Concurrently, the researcher recorded the method of implementing the care package in terms of compliance with infection control criteria, in the standard checklist. After catheterization, catheter care was daily evaluated using the second checklist. This assessment included checking hand washing 
before and immediately after contact with the catheter (before wearing gloves and after their removal), before and after changing the dressing, and while changing the dressing every three days by a sterile method in case of no discharges and regular inspection, until the catheter removal. All of these steps were done in the morning shift with the full presence and support of the researcher.

\section{Data analysis}

The samples were described using a set of frequency distribution tables and calculating the numerical indices. Also, the equality of the homogeneity of the variables in the two study groups was assessed using the independent $\mathrm{t}$ test and the chi-squared test, in SPSS V. 16.

\section{Results}

The comparison of the age, gender, and weight distribution of the neonates before and after the intervention indicated that the study groups were homogeneous in terms of these variables with no significant differences (Table 1).
The results of the catheter tip culture and blood infection in the neonates before and after the application of the care package confirmed the significant decrease of pathogens in the catheter tip $(\mathrm{P}=0.036)$ (Table 2). On the other hand, the catheter-associated bloodstream infection did not significantly differ before and after the implementation of the care package.

\section{Discussion}

According to the present results, staff training and the implementation of a standard care package significantly reduced the incidence of catheter infections. This is in line with the results obtained by Bannatyne et al., regarding the effects of a care package on reducing the incidence of central catheter-related infections at Canberra Hospital (Australia) (Bannatyne et al., 2018). The abovementioned study revealed that implementing the care package could significantly alter the clinical performance of using a central catheter, also, the change in the protocol decreased the clinical complications of the central catheter (e.g. reduced infection rate). The mentioned study also indicated a significant reduction of the central catheterassociated infection in the LBW infants, except for those

Table 1. Distribution of the demographic characteristics of neonates before and after presenting care package to catheter insertion team

\begin{tabular}{|c|c|c|c|c|}
\hline \multirow{2}{*}{ Group } & \multirow[b]{2}{*}{ Variables } & \multicolumn{3}{|c|}{ No. (\%) } \\
\hline & & Before Intervention & After Intervention & Test Results \\
\hline \multirow{3}{*}{ Gender } & Male & 27 (50.9) & $38(48.7)$ & $x^{2}=0.063$ \\
\hline & Female & $26(49.1)$ & $40(51.3)$ & $d f=1$ \\
\hline & Total & $53(100)$ & 78 (100) & $P=0.803$ \\
\hline \multirow{5}{*}{ Weight, g } & $1650-1990$ & $8(15.1)$ & $13(16.7)$ & \multirow{5}{*}{$\begin{array}{c}t=-1.286 \\
d f=129.000 \\
P=0.201\end{array}$} \\
\hline & 2000-2499 & $41(77.4)$ & $63(80.8)$ & \\
\hline & 2500 & $4(7.5)$ & $2(2.6)$ & \\
\hline & Total & $53(100)$ & $78(100)$ & \\
\hline & Mean $\pm S D$ & $2244 \pm 184.13$ & $2186.66 \pm 207.24$ & \\
\hline \multirow{7}{*}{ Age, day } & $\geq 1$ & $19(35.8)$ & $37(47.4)$ & \multirow{7}{*}{$\begin{array}{c}t=1.672 \\
d f=120.003 \\
P=0.097\end{array}$} \\
\hline & 2 & $24(45.3)$ & $18(23.1)$ & \\
\hline & 3 & $7(13.2)$ & $7(9.0)$ & \\
\hline & & & & \\
\hline & $<3$ & $3(5.7)$ & $16(20.5)$ & \\
\hline & Total & $53(100)$ & $78(100)$ & \\
\hline & Mean $\pm S D$ & $1.88 \pm 2.09$ & $4.57 \pm 2.95$ & \\
\hline
\end{tabular}


Table 2. Frequency distribution and the comparison of the catheter tip culture of neonates before and after care package training

\begin{tabular}{cccc}
\hline Group & $\begin{array}{c}\text { Pathogens in Culture of } \\
\text { Catheter Tip }\end{array}$ & Before Intervention & After Intervention \\
\hline Yes & $18(34.0)$ & $14(17.9)$ \\
No & $35(66.0)$ & $64(82.1)$ \\
Total & $53(100)$ & $78(100)$ \\
Chi-squared test results & & $P=0.036 ; \mathrm{df}=1 ; \mathrm{X}^{2}=4.38$ \\
\hline
\end{tabular}

Client- Centered Nursing Care

weighing 750 to 1000 grams. Therefore, although patients with low gestational age and low birth weight are at a higher risk of central line-related infection, implementing a care package significantly affects the patients admitted to the NICU. Besides, the significant increase in central line-related infections in neonates weighing 750 to 1000 grams is inconsistent with similar studies performed on a larger scale. The authors argued that ongoing studies were essential to reduce infections through the implementation of proper care packages, which could significantly impact each healthcare center and be extended to other centers for a wider effect. This goal could be achieved through ongoing training and assessment as well as innovations toward new interventions to further diminish neonatal infections (Bannatyne et al., 2018).

In another study, O'Neil et al. developed, implemented, and evaluated a central catheter care package to optimize central catheter maintenance techniques and reduce the associated infections in the patients admitted to the nonNICU wards of a large medical center affiliated with a university. After the intervention, the incidence of bacteremia-related infections in the intervention group decreased by $2.5 \%$ per month, although it was not significant (O'neil et al., 2016). The comparison of our findings with the results of the mentioned study indicated that adherence to this method for the care of an inserted central line could prevent catheter infections. Consistent with the present study, other findings have also highlighted the importance of central line maintenance methods in the prevention of catheter-related infections. These findings imply that all nationally published programs and guidelines on quality improvement and assurance should encompass these care recommendations for catheterization. Some of these guidelines include the training of the medical staff responsible for catheter maintenance, the disinfection of hubs, needleless connections, injection ports before catheter access, using alcohol and chlorhexidine to disinfect the skin, and regularly changing clear dressings (every 5-7 days) and light gauze dressings (every 2 days or whenever the dressing is contaminated, loose, or wet) (O'neil et al., 2016). Other consistent findings with the results of the present study also confirm (Sun et al., 2020; Tan et al., 2016; Resende et al., 2015).

The findings of the current research are congruent with a review study conducted by Payne et al. This study examined 24 quasi-experimental and observational studies regarding the implementation of a care package and its impact on the insertion of central lines at NICUs. The obtained results demonstrated a significant reduction in catheter-associated blood infection (Payne et al., 2018).

In the current research, the mean shelf life of the peripheral central venous catheter in the hospitalized neonates did not significantly differ before and after the intervention. Given the fact that LBW infants reach full intestinal nutrition later because of their immature gastrointestinal tract, this condition naturally prolongs the time they require central venous catheterization. As mentioned earlier, intravenous feeding is the foremost requirement of infants with a central catheter. In this regard, our findings could also depend on the medical judgment as to whether or not the catheter should be removed. For example, the catheter works but is removed since the infant does not need a catheter. Greenberg et al. tested the prolonged catheterization and its association with the higher incidence of central catheter infections. The findings indicated that central catheter infections were not associated with the shelf life. The mentioned study stated that the medical team should focus their efforts on reducing the risk of central catheter infections as well as the proper maintenance and timely removal of catheters (Greenberg et al., 2015).

In the study by Butler-O'Hara et al., the effects of implementing a care package were evaluated during catheter insertion on increasing the shelf life, and significant findings were reported (Butler-O'Hara et al., 2012). In another study performed in China, shelf life was observed to increase significantly after using a care package, which differed from the present study (Cheng et al., 2016). The discrepancy between the results of the 
present study and the mentioned studies may be due to other factors affecting the durability of a catheter.

According to the literature, several factors affect the longevity of a catheter, and the longevity may vary in the premature infants admitted to the NICU (Li et al., 2019). The judgment of the medical team based on the clinical condition of the neonates affects the removal or persistence of the catheter. Therefore, the provision of a care package is not the only influential factor in the shelf life of a catheter. In the present study, most of the variables affecting the shelf life of the catheters were matched, and the samples were homogeneous in this regard. Catheter durability can be affected by several factors, such as complications during and after catheter insertion, misplacement/displacement of the catheter, and catheter care and maintenance. Thus, there are complex cases and potential complications associated with PICC insertion, which require proper management.

\section{Conclusion}

Considering the results of the present study and the positive effects of using a care package on the reduction of central venous catheter infections in preterm infants, further investigations are suggested to assess the educational needs of the catheter implantation team and compare the performance of neonatal wards with and without a specific team for catheter implantation. Furthermore, it is recommended to evaluate other methods to prevent the possible complications of catheterization in infants. For instance, the differences between the effects of catheterization, ultrasonography, and conventional methods on the reduction of neonatal hypothermia could be studied.

To control the accurate implementation of the care package, the evaluation was performed using a checklist with the help of the coresearcher and the catheter insertion team, in the fixed morning shift. Therefore the nurses' self-assessment by the checklist in other shifts might have affected the results. To resolve this issue, the researcher attempted to unify the assessment across all the shifts by training all the personnel. Nevertheless, complete control of this situation was beyond the researcher's control. Another limitation of our study was the decision to remove the catheter when the infant did not need one despite the efficiency of the catheter, which might have affected the shelf life measurement of the catheter.

\section{Ethical Considerations}

\section{Compliance with ethical guidelines}

This study was approved by the Bioethics Committee of Iran University of Medical Sciences (Code: IR.IUMS. REC.1397.777). Written informed consent was obtained from the parents of the neonates who participated in the study.

\section{Funding}

This article was extracted from a MA. thesis of the first author at the Department of Pediatric Nursing, School of Nursing and Midwifery, Iran University of Medical Sciences, Tehran

\section{Authors' contributions}

Conceptualization, methodology: Mohammad Kazemian; Methodology investigation, resources, data curation: Fariba Safaee; Writing - original draft preparation, supervision, project administration: Leili Borimnejad; Writing - review \& editing, visualization: Marjan Rasouli.

\section{Conflict of interest}

The authors declared no conflict of interest.

\section{Acknowledgments}

Hereby, we extend our gratitude to the Iran University of Medical Sciences for the financial support of this study. We would also like to thank Shahid Beheshti University of Medical Sciences, especially the Staff of the intensive Care Unit of Teaching Hospital for assisting us in this research project.

\section{References}

Alexandrou, E., et al., 2018. Use of short peripheral intravenous catheters: Characteristics, management, and outcomes worldwide. Journal of Hospital Medicine, 13(5), pp. E1-E7. [DOI:10.12788/jhm.3039] [PMID]

Bannatyne, M., et al., 2018. Retrospective cohort analysis of central line associated blood stream infection following introduction of a central line bundle in a neonatal intensive care unit. International Journal of Pediatrics, 2018, p. 4658181. [DOI:10.1155/2018/4658181] [PMID] [PMCID]

Baranowski, S. T., Hummler, H., \& Hopfner, R. J., 2014 Unusual malposition of a Peripherally Inserted Cen- 
tral Catheter (PICC) in a Very Low Birth Weight infant (VLBW Infant). Klin Padiatr, 226(4), pp. 226-48. [DOI:10.1055/s-0034-1376948] [PMID]

Butler-O'Hara, M., et al., 2012. An evidence-based catheter bundle alters central venous catheter strategy in newborn infants. The Iournal of Pediatrics, 160(6), pp. 972-77. [DOI:10.1016/j.jpeds.2011.12.004] [PMID]

Cheng, H. Y., et al., 2016. Increased frequency of peripheral venipunctures raises the risk of central-line associated bloodstream infection in neonates with peripherally inserted central venous catheters. Journal of Microbiology, Immunology and Infection, 49(2), pp. 230-6. [DOI:10.1016/j.jmii.2014.06.001] [PMID]

Cotogni, P., et al., 2015. Peripherally inserted central catheters in non-hospitalized cancer patients: 5-year results of a prospective study. Supportive Care in Cancer, 23(2), pp. 403-9. [DOI:10.1007/s00520-014-2387-9] [PMID] [PMCID]

Emoli, A., et al., 2014. [The ISP (Safe Insertion of PICCs) protocol: a bundle of 8 recommendations to minimize the complications related to the Peripherally Inserted Central Venous Catheters (PICC) (Ita)]. Assistenza Infermieristica e Ricerca, 33(2), pp. 82-9. [DOI:10.1702/1539.16813] [PMID]

Gilbert, R., et al., 2019. Antimicrobial-impregnated central venous catheters for Prevention of Neonatal Bloodstream Infection (PREVAIL): An open-label, parallel-group, pragmatic, randomised controlled trial. The Lancet Child \& Adolescent Health, 3(6), pp. 381-90. [DOI:10.1016/S2352-4642(19)30114-2]

Gorski, L. A., 2017. The infusion therapy standards of practice. Home Healthcare Now, 35(1), pp. 10-18. [DOI:10.1097/ NHH.0000000000000481] [PMID]

Greenberg, R. G., et al., 2015. Effect of catheter dwell time on risk of central line-associated bloodstream infection in infants. Pediatrics, 136(6), pp. 1080-6. [DOI:10.1542/peds.20150573] [PMID] [PMCID]

Li, R., et al., 2019. Application of peripherally inserted central catheters in critically ill newborns experience from a neonatal intensive care unit. Medicine (Baltimore), 98(32), p. e15837. [DOI:10.1097/MD.0000000000015837] [PMID] [PMCID]

Martín-Rabadán, P., et al., 2017. Improved method for the detection of catheter colonization and catheter-related bacteremia in newborns. Diagnostic Microbiology and Infectious Disease, 87(4), pp. 311-4. [DOI:10.1016/j.diagmicrobio.2017.01.001] [PMID]

O'neil, C., et al., 2016. A central line care maintenance bundle for the prevention of central line-associated bloodstream infection in non-intensive care unit settings. Infect Control Hosp Epidemiol, 37(6), pp. 692-8. [DOI:10.1017/ice.2016.32] [PMID] [PMCID]

Payne, V., et al., 2018. Care bundles to reduce central lineassociated bloodstream infections in the neonatal unit: A systematic review and meta-analysis. Archives of Disease in Childhood - Fetal and Neonatal Edition, 103(5), pp. F422-F429. [DOI:10.1136/archdischild-2017-313362] [PMID]

Resende, D. S., et al., 2015. Late onset sepsis in newborn babies: epidemiology and effect of a bundle to prevent central line associated bloodstream infections in the neonatal intensive care unit. Brazilian Journal of Infectious Diseases, 19(1), pp. 527. [DOI:10.1016/j.bjid.2014.09.006] [PMID]
Rosenthal, V. D., et al., 2020. International Nosocomial Infection Control Consortium (INICC) report, data summary of 45 countries for 2012-2017: Device-associated module. American Journal of Infection Control, 48(4), pp. 423-32. https://www.sciencedirect.com/science/article/abs/pii/ S0196655319307953

Santhakumaran, S., et al., 2018. Survival of very preterm infants admitted to neonatal care in England 2008-2014: Time trends and regional variation. Archives of Disease in Childhood - Fetal and Neonatal Edition, 103(3), pp. F208-F215. [DOI:10.1136/ archdischild-2017-312748] [PMID] [PMCID]

Sharpe, E., et al., 2017. Neonatal peripherally inserted central catheter practices and providers: Results from the neonatal PICC1 Survey. Advances in Neonatal Care: Official Journal of the National Association of Neonatal Nurses, 17(3), pp. 209-21. [DOI:10.1097/ANC.0000000000000376] [PMID]

Sun, Y., et al., 2020. Positive effect of care bundles on patients with central venous catheter insertions at a tertiary hospital in Beijing, China. Journal of International Medical Research, 48, p. 0300060520942113. [DOI:10.1177/0300060520942113] [PMID] [PMCID]

Tan, J., et al., 2016. Cost-effectiveness analysis of ultrasoundguided Seldinger Peripherally Inserted Central Catheters (PICC). Springerplus, 5(1), pp. 1-10. [DOI:10.1186/s40064016-3698-8] [PMID] [PMCID]

Wang, W., et al., 2015. Prevention of peripherally inserted central line-associated blood stream infections in very low-birthweight infants by using a central line bundle guideline with a standard checklist: a case control study. BMC Pediatrics, 15, $\mathrm{p}$. 69. [DOI:10.1186/s12887-015-0383-y] [PMID] [PMCID] 